Electric-field induced ferromagnetic domain changes in exchange biased $\mathrm{Co}-\mathrm{BiFeO}_{3}$ composites

This article has been downloaded from IOPscience. Please scroll down to see the full text article.

2010 J. Phys. D: Appl. Phys. 43165001

(http://iopscience.iop.org/0022-3727/43/16/165001)

View the table of contents for this issue, or go to the journal homepage for more

Download details:

IP Address: 143.248.16.189

The article was downloaded on 16/06/2010 at 02:28

Please note that terms and conditions apply. 


\title{
Electric-field induced ferromagnetic domain changes in exchange biased $\mathrm{Co}-\mathrm{BiFeO}_{3}$ composites
}

\author{
Sang-Hyun Kim ${ }^{1}$, Hyunwoo Choi ${ }^{2}$, Kwangsoo $\mathrm{No}^{2}$ and Sung-Chul Shin ${ }^{1}$ \\ ${ }^{1}$ Department of Physics and Center for Nanospinics of Spintronic Materials, Korea Advanced Institute \\ of Science and Technology, Daejeon 305-701, Korea \\ ${ }^{2}$ Department of Material Science and Engineering, Korea Advanced Institute of Science and \\ Technology, Daejeon 305-701, Korea
}

Received 2 November 2009, in final form 28 February 2010

Published 8 April 2010

Online at stacks.iop.org/JPhysD/43/165001

\begin{abstract}
We present direct observations of electric-field induced changes in the local ferromagnetic (F) domain structure in films formed from inclusions of $\mathrm{F}$ cobalt in an antiferromagnetic host of $\mathrm{BiFeO}_{3}$. Piezoelectric force microscopy was used to demonstrate that the composite $\mathrm{Co}-\mathrm{BiFeO}_{3}$ films display ferroelectricity. Strikingly, magnetic contrast changes in magnetic force microscopic images were found with the application of a perpendicular electric field. These are the results of an exchange interaction between $\mathrm{Co}$ and $\mathrm{BiFeO}_{3}$.

(Some figures in this article are in colour only in the electronic version)
\end{abstract}

\section{Introduction}

Manipulation of the magnetization by an electric field is promising for the development of spintronic devices [1-3]. For this reason there has been considerable recent interest in magnetoelectric multiferroics. Among these, $\mathrm{BiFeO}_{3}$ (BFO) is the only room-temperature single-phase multiferroic. BFO exhibits a ferroelectric Curie temperature $\left(T_{\mathrm{C}}\right)$ of $\sim 1100 \mathrm{~K}$ and an antiferromagnetic Néel temperature $\left(T_{\mathrm{N}}\right)$ of $\sim 640 \mathrm{~K}$ $[4,5]$. Microscopically, BFO displays an incommensurate antiferromagnetic ordering with a cycloidal wavelength $\lambda \sim$ $600 \AA$ [6]. It has been shown that a strong coupling between ferroelectricity and antiferromagnetism (AF) takes place in BFO: the antiferromagnetic easy-plane is changed by switching the direction of the ferroelectric polarization $[7,8]$. This effect has been used in CoFe/BFO bilayers to control the direction of the magnetization of the ferromagnetic $(F)$ CoFe layer via electric-field control of the exchange bias at the interface between the $\mathrm{AF} \mathrm{BFO}$ and the $\mathrm{F} \mathrm{CoFe}$ thin film layers [3]. In this paper, we present direct observation of electric-field induced local $\mathrm{F}$ domain changes in $\mathrm{Co}-\mathrm{BFO}$ composite films at room temperature through exchange interaction between ferromagnet $\mathrm{Co}$ and antiferromagnet BFO.

\section{Experimental procedure}

Co-BFO composite films, $800 \mathrm{~nm}$ thick, were grown on $\left(\begin{array}{lll}0 & 0 & 1\end{array}\right)$ $\mathrm{SrTiO}_{3}$ (STO) substrates using sputtering in $30 \mathrm{mTorr}$ pure Ar. A Bi-rich $\mathrm{Bi}_{1.1} \mathrm{FeO}_{3}$ target was used to compensate for the high vapour pressure of $\mathrm{Bi}$ at the substrate temperature of $600{ }^{\circ} \mathrm{C}$. Co chips were placed on the $\mathrm{Bi}_{1.1} \mathrm{FeO}_{3}$ target to provide for Co doping. To characterize the electrical properties of the films, a $100 \mathrm{~nm}$ thick $\mathrm{SrRuO}_{3}$ layer was deposited on the STO substrate to form a lower conducting electrode. Co concentration was analysed by an energy dispersive spectrometer (EDS). The crystal structure of the films was characterized by $\mathrm{x}$-ray diffractometry (XRD). The magnetic properties were measured using an alternating gradient force magnetometer (AGM). Magnetic images were obtained by magnetic force microscopy (MFM). Piezoelectric response data were acquired by piezoelectric force microscopy (PFM). All experiments were conducted at room temperature.

\section{Results and discussion}

Figure 1 shows a $\theta-2 \theta$ XRD scan of a $\mathrm{Co}_{x}-(\mathrm{BFO})_{1-x}$ film with $x=0.038$ where $x=0.038$ means 3.8 atomic\% of Co. 


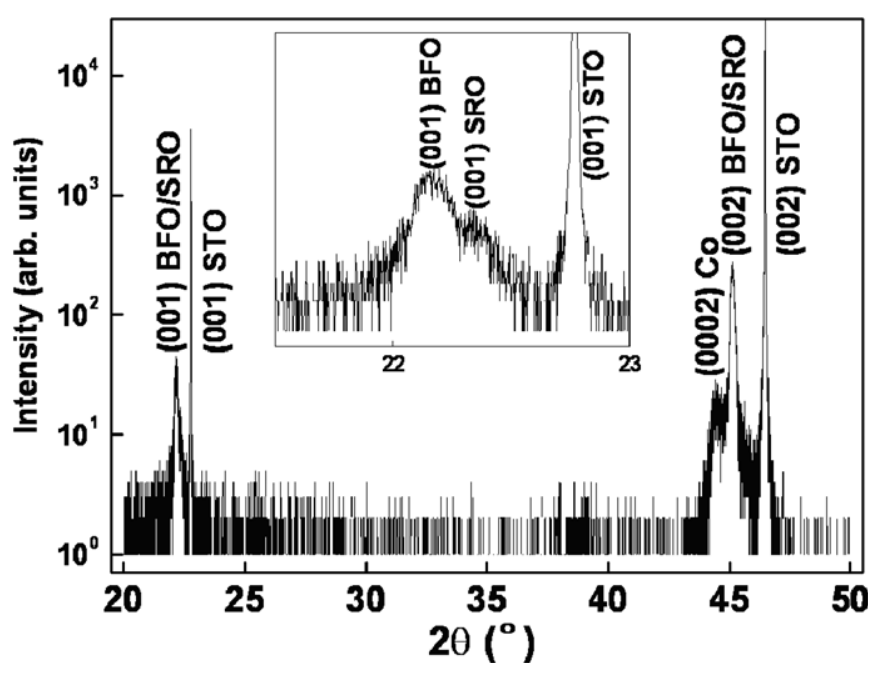

Figure 1. X-ray $\theta-2 \theta$ scan of $800 \mathrm{~nm}$ thick $\mathrm{Co}-\mathrm{BiFeO}_{3}$ film deposited on (0 01 1) $\mathrm{SrRuO}_{3} / \mathrm{SrTiO}_{3}$ substrate. The three $\left(\begin{array}{lll}0 & 0 & 1\end{array}\right)$ $\mathrm{BFO} / \mathrm{SRO} / \mathrm{STO}$ peaks are shown enlarged in the inset.

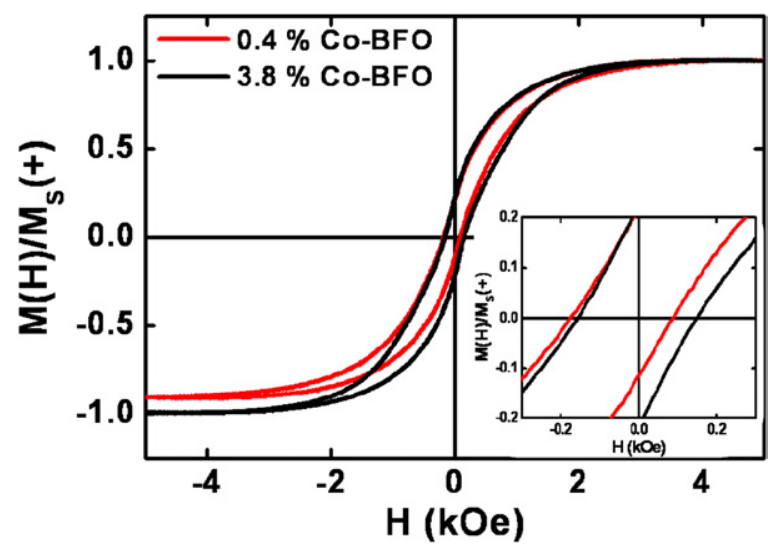

Figure 2. $M-H$ hysteresis loops of $800 \mathrm{~nm}$ thick $\mathrm{Co}-\mathrm{BFO}$ films on ( 001 ) $\mathrm{SrTiO}_{3}$ substrates. The Co concentration (atomic per cent) is shown in the figure. The inset shows a magnified view of the low field data.

Only peaks corresponding to the $(00 l)$ peaks of BFO and the $\left(\begin{array}{lll}0 & 0 & 2\end{array}\right)$ peak of hcp Co were found showing that the film is highly $\left(\begin{array}{lll}0 & 0 & 1\end{array}\right)$ textured. No evidence for the formation of any oxide of $\mathrm{Co}, \mathrm{Bi}, \mathrm{Fe}$ or $\mathrm{Co}$ ferrite was found.

Figure 2 shows a comparison of the magnetization versus in-plane field $(M-H)$ hysteresis loops of two Co-BFO films measured at room temperature with $x=0.004$ and 0.038 . The two $M-H$ loops were normalized by the saturation magnetization in the positive field $\left(M_{\mathrm{S}}(+)\right)$. The inset in figure 2 clearly shows a loop shift to the negative field direction $(-88 \mathrm{Oe})$ and a coercivity enhancement of the Co-BFO films. Such behaviour related to the exchange bias effect could be explained using the intuitive model proposed by Meiklejohn and Bean $[9,10]$. Given the exchange interaction between the BFO and Co interfaces, if the coupling between the uncompensated spins in the BFO film (with the rest of the $\mathrm{BFO}$ film) is strong enough, the AF BFO will not change its magnetic alignment at low fields and pin the F Co, so there will be an exchange bias. On the other hand, if there is more
Co in the sample, the coupling between the BFO and Co spins could become stronger than the one between the spins within $\mathrm{BFO}$, so it is energetically more favourable that the spins at the $\mathrm{BFO} / \mathrm{Co}$ interface rotate together, contributing to the coercivity enhancement, not to the exchange bias. The other interesting point is the fact that the $M-H$ loop with $x=0.004$ shifts upwards, i.e. the magnitude of the saturation magnetization in the positive field direction is larger than that in the negative field direction. Such a vertical shift tied in with the existence of exchange bias field has been known to arise from the pinned uncompensated antiferromagnetic spins at the interface between antiferromagnet and ferromagnet $[11,12]$. Therefore, the observed vertical shift in this system implies the existence of the pinned uncompensated BFO spins in the vicinity of the embedded Co particles. It is worth mentioning that the exchange bias is witnessed without an intentional field cooling. The stray field of $\sim 30$ Oe from a magnetron sputtering gun during film fabrication seems to play a role [13, 14]. One might postulate that the exchange bias arises from the formation of antiferromagnetic cobalt oxide even though this was not detected by XRD. However, no exchange bias field has been observed in oxide passivated Co particles at $300 \mathrm{~K}[15,16]$. Moreover, similar Co-STO films $(x=0.005$ and 0.043$)$ grown on (0 0 1) STO substrates under the same deposition conditions as the Co-BFO films exhibit no exchange bias, but the constant coercivity of $50 \mathrm{Oe}$ [17].

The coexistence of ferroelectricity and magnetism in our Co-BFO films was revealed by PFM and MFM studies. Figures 3(a) and $(b)$ compare an out-of-plane phase PFM image of a $\mathrm{Co}_{x}-(\mathrm{BFO})_{1-x}(x=0.038)$ film with that of a pure BFO film. Firstly, a square region of $\sim 5 \times 5 \mu \mathrm{m}^{2}$ was written at $+10 \mathrm{~V}$ and the central $\sim 2 \times 2 \mu \mathrm{m}^{2}$ area was written at the opposite voltage of $-10 \mathrm{~V}$. Then the images shown in figures 3(a) and $(b)$ were taken. These correspond to an area of $\sim 4 \times 4 \mu \mathrm{m}^{2}$. The images clearly show the oppositely written regions establishing that both $\mathrm{Co}-\mathrm{BFO}$ and $\mathrm{BFO}$ films support ferroelectricity. Note that the pure BFO film is of almost uniform contrast, whereas the Co-BFO film clearly shows structure in both bright and dark areas, which might be attributed to Co inclusions since Co is not ferroelectric.

We use MFM to explore the possibility of $\mathrm{F}$ inclusions of $\mathrm{Co}$ in the Co-BFO films. Indeed, MFM reveal magnetic contrast as shown in the images in figures $3(c)$ and $(d)$, which were taken in $H=0$ after applying fields of $+15 \mathrm{kOe}$ and $-15 \mathrm{kOe}$, respectively. Note that the sample was removed from the MFM to apply the field and then the same area was located. One can clearly see that predominantly the dark spots in figure 3(c) are changed into bright spots by reversing the magnetic field. Therefore, we conclude that the dark and bright contrasts correspond to oppositely magnetized Co dispersed in the BFO film.

To investigate electric-field induced magnetization changes, MFM images of the same area of the Co-BFO film were taken in sequence after (a) application of a magnetic field of $+15 \mathrm{kOe}$, (b) electrical poling at $+12 \mathrm{~V}$, (c) electrical poling at $-12 \mathrm{~V}$ and (d) electrical poling at $+12 \mathrm{~V}$, as shown in figures $4(a)-(d)$. Note that a nonmagnetic conductive tip was used for electrical poling in contact mode, while a magnetic 

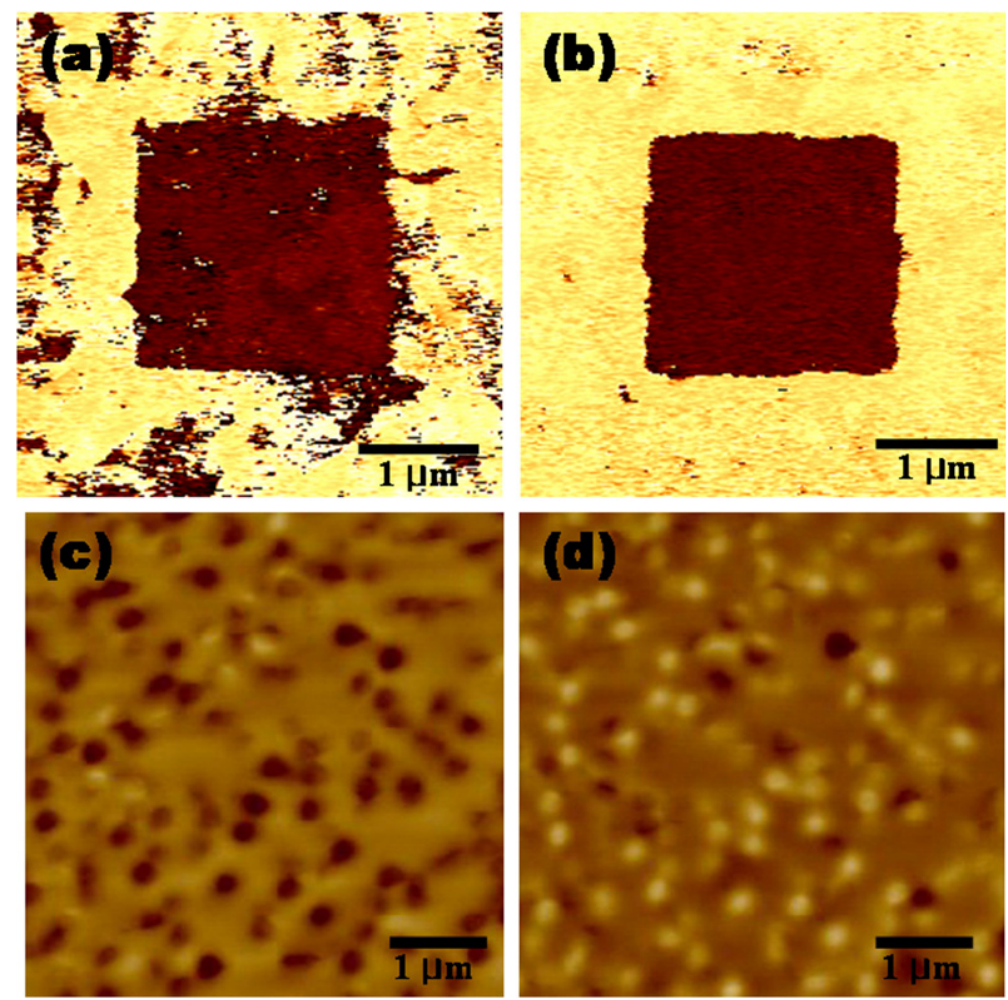

Figure 3. Out-of-plane phase PFM image of (a) Co-BFO film and $(b)$ BFO film. MFM images of the identical Co-BFO film in $(a)$ after applying a magnetic field of $15 \mathrm{kOe}(b)$ up and $(c)$ down. The MFM images are from the same film area.
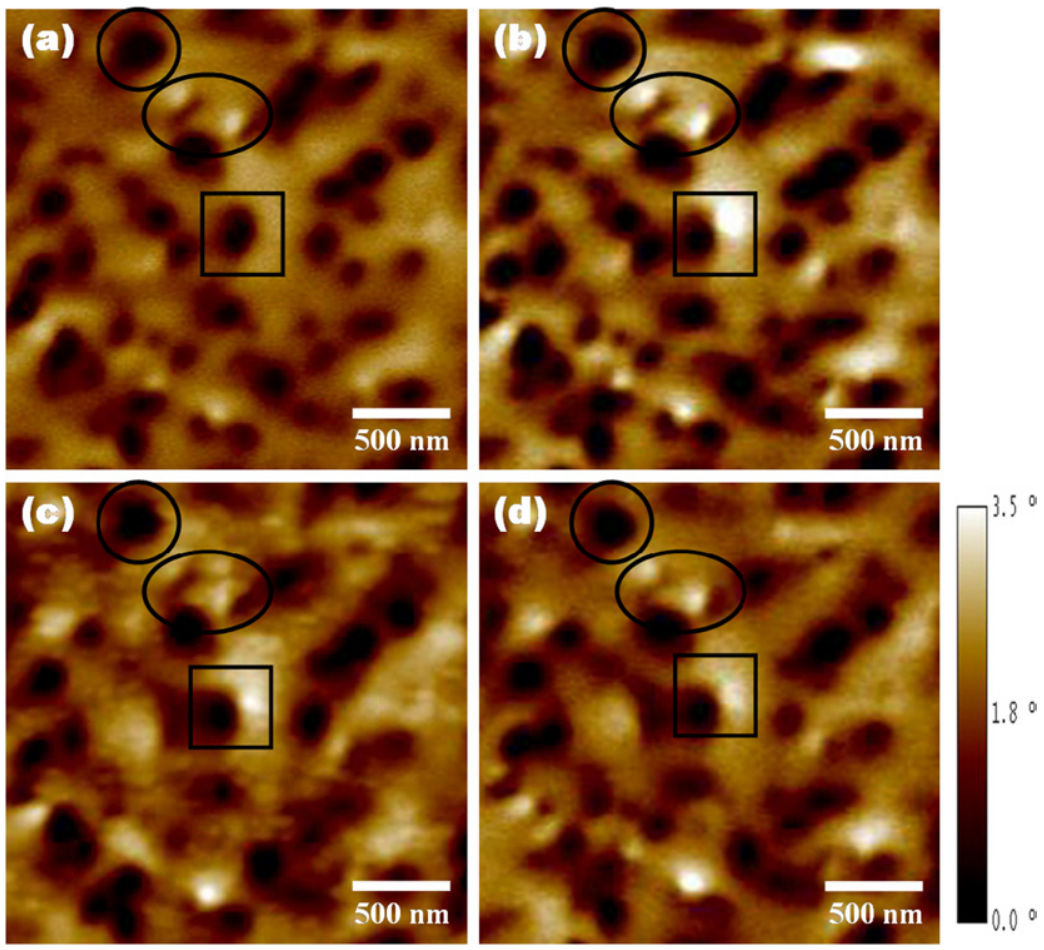

Figure 4. MFM images of the same area in the $\mathrm{Co}-\mathrm{BiFeO}_{3}$ film of figure 3 after $(a)$ magnetizing the film in $H=+15 \mathrm{kOe},(b)$ after a first electrical poling at $+12 \mathrm{~V},(c)$ after a second electrical poling at $-12 \mathrm{~V}$ and $(d)$ after a third electrical poling at $+12 \mathrm{~V}$.

tip was used for probing the magnetic domain images (using phase detection). After the first electrical poling, three types of changes were observed in the magnetic contrast of the MFM images: (i) dark contrast partly changed to bright (square), (ii) dark contrast unchanged (circle) and (iii) bright/dark contrast becoming brighter (ellipse). Since the dark and bright contrasts represent different directions of local magnetization with respect to the magnetic tip, observations of changes in 
the MFM image contrasts imply rotations of magnetization direction induced by the electric field.

The rotation of magnetization direction with the application of an electric field is believed to be due to the presence of two couplings: the exchange interaction between $\mathrm{Co}$ and $\mathrm{BFO}$ and a coupling between the ferroelectricity and $\mathrm{AF}$ of BFO. The ferroelectric polarization in $\mathrm{BFO}$ is aligned along the $\left\langle\begin{array}{lll}1 & 1 & 1\end{array}\right\rangle$ axes. Thus, in $\left(\begin{array}{lll}0 & 0 & 1\end{array}\right)$ BFO there are eight possible domains. Consequently, the orientation of the polarization can be switched by $71^{\circ}, 109^{\circ}$ and $180^{\circ}$ from its original orientation [18]. Since the moments in the antiferromagnetic phase are oriented in the plane perpendicular to the ferroelectric polarization direction, only changes in polarization direction by $71^{\circ}$ or $109^{\circ}$ will change the antiferromagnetic order in BFO. In addition, the change in the antiferromagnetic order induced by a $71^{\circ}$ polarization change is more complex than that of a $109^{\circ}$ change. For example, the sixfold degeneracy of the orientation of the antiferromagnetic sublattice magnetization within the (111) plane could be lifted in a strained BFO film that was proved by XRD reciprocal space mapping data of our (001) BFO film and the antiferromagnetic axis is aligned parallel to the $[1-10]$ direction [8]. As the $[1-10]$ direction is perpendicular to the [llll 111 direction, in the case of a $71^{\circ}$ polarization change where the polarization orientation rotates from [ 1111$]$ to $\left[\begin{array}{lll}1 & 1 & -1\end{array}\right]$, the antiferromagnetic axis is preserved, because the $\left[\begin{array}{ll}1 & 1-1\end{array}\right]$ direction is still perpendicular to $[1-10]$. However, in a $109^{\circ}$ polarization change where the polarization orientation switches from [ $\left[\begin{array}{lll}1 & 1 & 1\end{array}\right]$ to $\left[\begin{array}{lll}-1 & 1 & -1\end{array}\right]$, the antiferromagnetic axis rotates by $90^{\circ}$, because the $[-11-1]$ direction is not perpendicular to $[1-10]$. The rotation of the antiferromagnetic axis in $\mathrm{BFO}$ by the $109^{\circ}$ polarization change can give rise to a rotation of the Co magnetization direction through exchange interaction between ferromagnet Co and antiferromagnet BFO such as the areas indicated by the squares and the ellipses. It was also found that the bright contrasts in the areas in the squares and the ellipses of figure $4(b)$ became fainter with the electrical poling of $-12 \mathrm{~V}$ and bright again after the electrical poling of $+12 \mathrm{~V}$, as seen in figures $4(c)$ and (d), respectively.

On the other hand, no change of the antiferromagnetic axis in BFO by the $71^{\circ}$ or $180^{\circ}$ polarization switching means the absence of changes of magnetic domain contrasts such as the area indicated by the circles. The area in the circles remained unchanged during the entire processes. From the PFM images, the out-of-plane component of the polarization of the Co-( $\left.\begin{array}{lll}0 & 0 & 1\end{array}\right)$ BFO film was indeed shown to be switched on application of an electric field, implying the presence of those ferroelectric polarization switching mechanisms in the film. Therefore, we interpret the observed magnetic contrast changes in MFM images to be due to exchange interaction between ferromagnet $\mathrm{Co}$ and antiferromagnet $\mathrm{BFO}$, which is changed with an electric field.

We also consider elastic coupling between $\mathrm{Co}$ and BFO for the observed electric-field induced F domain changes [2]. The magnetoelastic energy density with an electrical poling of $12 \mathrm{~V}$ could be approximately $1.2 \times 10^{2} \mathrm{~J} \mathrm{~m}^{-3}$, which is calculated using $\lambda_{33} \sim 2 \times 10^{-6}, d_{33} \sim 40 \mathrm{pm} \mathrm{V}^{-1}$ at $15 \mathrm{MV} \mathrm{m}^{-1}$ and $Y \sim 100 \mathrm{GPa}$ [19-21]. The magnetic anisotropy energy density $E_{\text {ani }} \sim 5.4 \times 10^{3} \mathrm{~J} \mathrm{~m}^{-3}$, which is calculated from the $M-H$ loop (figure 2), is much larger than the magnetoelastic energy density $E_{\mathrm{me}} \sim 1.2 \times 10^{2} \mathrm{~J} \mathrm{~m}^{-3}$. Moreover, the MFM images were taken after removing an electric field. Therefore, we believe that the observed electric-field induced $\mathrm{F}$ domain changes are not attributed to elastic coupling between Co and BFO.

\section{Conclusion}

In summary, $\mathrm{Co}-\mathrm{BFO}$ composite films were grown on (00 01 ) STO substrates by co-sputtering of Co and BFO. Comparison of magnetization versus field loops with those from Co-STO films shows evidence for a loop shift and a coercivity enhancement which we conclude from the exchange interaction between $\mathrm{Co}$ and BFO. The BFO was found to be ferroelectric from PFM. Magnetic contrast changes were observed in MFM images with the application of an electric field, directly providing evidence of an electric-field induced change in the $\mathrm{F}$ domain structure. We ascribe these changes to the exchange interaction between Co and BFO and the coupling between ferroelectricity and the AF of BFO.

\section{Acknowledgments}

This work was supported by the National Research Laboratory Programme (R0A-2007-000-20026-0), the Global Partnership Programme (K20702000014-08E0200-01410) and the World Class University Programme (R33-2008-00010078-0) through the National Research Foundation of Korea funded by the Ministry of Education, Science and Technology.

\section{References}

[1] Chiba D, Sawicki M, Nishitani Y, Nakatani Y, Matsukura F and Ohno H 2008 Nature 455515

[2] Zavaliche F et al 2005 Nano Lett. 51793

[3] Chu Y H et al 2008 Nature Mater. 7478

[4] Kiselev S V, Ozerov R P and Zhdanov G S 1963 Sov. Phys.-Dokl. 7742

[5] Teague J R, Gerson R and James W J 1970 Solid State Commun. 81073

[6] Sosnowska I, Peterlin-Neumaier T and Steichele E 1982 J. Phys. C. Solid State Phys. 154835

[7] Ederer C and Spaldin N A 2005 Phys. Rev. B 71 060401(R)

[8] Zhao T et al 2006 Nature Mater. 5823

[9] Meiklejohn W H 1962 J. Appl. Phys. 331328

[10] Nogues J and Schuller I K 1999 J. Magn. Magn. Mater. 192203

[11] Nogués J, Sort J, Langlais V, Skumryev V, Surinach S, Munoz J S and Baro M D 2005 Phys. Rep. 42265

[12] Zheng R K, Wen G H, Fung K K and Zhang X X 2004 J. Appl. Phys. 955244

[13] Garcia F, Moritz J, Ernult F, Auffret S, Rodmacq B, Dieny B, Camarero J, Pennec Y, Pizzini S and Vogel J 2002 IEEE Trans. Magn. 382730

[14] Liu Z Y and Adenwalla S 2003 Appl. Phys. Lett. 822106

[15] Skumryev V, Stoyanov S, Zhang Y, Hadjipanayis G, Givord D and Nogués J 2003 Nature $\mathbf{4 2 3} 850$ 
[16] Peng D L, Sumiyama K, Hihara T, Yamamuro S and Konno T J 2000 Phys. Rev. B 613103

[17] Kim S H, Kim K Y and Shin S C J. Appl. Phys. submitted

[18] Zavaliche F, Yang S Y, Zhao T, Chu T H, Cruz M P, Eom C B and Ramesh R 2006 Phase Transit. 79991

[19] Bozorth R M 1954 Phys. Rev. 96311
[20] Wang J, Neaton J B, Zheng H, Nagarajan V, Ogale S B, Liu B, Spaldin N A, Rabe K M, Wuttig M and Ramesh R 2003 Science 2991719

[21] Kounga Njiwa A B, Aulbach E, Rodel J, Turner S L, Comyn T P and Bell A J 2006 J. Am. Ceram. Soc. 891761 